\title{
Area Under the Urinary Excretion Rate Curve from T1 to T2
}

National Cancer Institute

\section{Source}

National Cancer Institute. Area Under the Urinary Excretion Rate Curve from T1 to T2. NCl Thesaurus. Code C85572.

The area under the urinary excretion rate curve (AURC) from T1 to T2. 